\title{
Análise sobre a forma da escrita conclusiva em artigos científicos na área de Ciências
}

\section{Agrárias}

\author{
Analysis on the form of conclusive writing in scientific articles in the field of Agrarian Sciences
}

Análisis sobre la forma de redacción concluyente en artículos científicos en el campo de las Ciencias

Agrícolas

Recebido: 23/04/2021 | Revisado: 03/05/2021 | Aceito: 04/05/2021 | Publicado: 17/05/2021

Italo de Souza Aquino

ORCID: https://orcid.org/0000-0002-7948-8760 Universidade Federal da Paraíba, Brasil italo.aquino@terra.com.br

\begin{abstract}
Resumo
Conclusão é o último elemento textual em um artigo científico; sua forma de apresentação, porém, ainda não é padronizada entre as publicações de revistas científicas. O objetivo desta investigação foi efetuar uma análise sobre a forma da escrita conclusiva em artigos científicos nacionais, com base em 40 periódicos da área de ciências agrárias, publicados no período 2003-2017. Os indicadores analisados foram: formas (sinônimos) da escrita de Conclusão; tipo de sinal usado antes de cada conclusão; número de conclusões; estilo da conclusão; tempo de verbo utilizado nos parágrafos conclusivos; presença de expressões personificadas; e presença do objeto de estudo nos parágrafos conclusivos. Os resultados demonstram que a expressão CONCLUSÕES (plural e em caixa alta) é a mais usada $(32,507 \%)$ entre as revistas analisadas; o uso de parágrafo simples (sem número ou símbolo) é predominante (70\%); 45\% dos artigos apresentam 2 (duas) conclusões específicas; 70\% dos artigos exibem apenas a Conclusão (sem informação adicional); o tempo de verbo no "presente" é a forma mais utilizada nos parágrafos conclusivos (40\%); as conclusões são escritas de forma impessoal (100\%); e a presença do objeto de estudo nos parágrafos conclusivos tem maior prevalência através da nomenclatura vulgar (62,50\%). Conclui-se que a forma diversificada de apresentação dos parágrafos conclusivos em artigos científicos nacionais, na área de ciências agrárias, merece uma atenção especial na busca de um modelo que permita estreitar sua múltipla forma para uma escrita mais funcional.
\end{abstract}

Palavras-chave: Ensino; Metodologia científica; Conclusão; Artigo científico.

\begin{abstract}
Conclusion is the last textual element in a scientific paper; Its presentation form, however, is not yet standardized among the publications of scientific journals. The objective of this research was to carry out an analysis on the form of the conclusive writing in national scientific articles, based on 40 periodicals of the area of agrarian sciences, published in the period 2003-2017. The analyzed indicators were: forms (synonyms) of the writing of Conclusion; Type of signal used before each conclusion; Number of conclusions; Style of the Conclusion; Verb tense used in the concluding paragraphs; Presence of personifying expressions; and presence of the object of study in the concluding paragraphs. The results show that the expression CONCLUSIONS (plural and upper case) is the most used (32.507\%) among the analyzed journals; Simple paragraph (without number or symbol) is predominant $(70 \%)$; $45 \%$ of the articles present 2 (two) specific conclusions; $70 \%$ of the articles only show Conclusion (without additional information); The "present" tense is the most used form in the concluding paragraphs (40\%); conclusions are written impersonally (100\%); and the presence of the object of study (in all articles: crops) in the concluding paragraphs is more prevalent through vulgar nomenclature (62.50\%). It is concluded that the diverse form of presentation of the conclusive paragraphs in national scientific articles, in the area of agriculture sciences, deserves special attention in the search for a model that allows narrowing its multiple form in order to achieve a more functional writing.
\end{abstract}

Keywords: Teaching; Scientific methodology; Conclusion; Scientific article.

\section{Resumen}

La conclusión es el último elemento textual de un artículo científico; su forma de presentación, sin embargo, aún no está estandarizada entre las publicaciones de revistas científicas. El objetivo de esta investigación fue realizar un análisis sobre la forma de redacción concluyente en artículos científicos nacionales, a partir de 40 revistas del área de ciencias agrícolas, publicadas en el período 2003-2017. Los indicadores analizados fueron: formas (sinónimos) de redacción de Conclusiones; tipo de señal utilizada antes de cada conclusión; número de conclusiones; estilo de terminación; tiempo verbal usado en los párrafos finales; presencia de expresiones personificadas; y presencia del objeto de estudio en los párrafos finales. Los resultados muestran que la expresión CONCLUSIONES (plural y 
mayúscula) es la más utilizada (32,507\%) entre las revistas analizadas; predomina el uso de un párrafo simple (sin número ni símbolo) (70\%); El 45\% de los artículos presenta 2 (dos) conclusiones específicas; El 70\% de los artículos muestran solo la Conclusión (sin información adicional); el tiempo verbal en "presente" es la forma más utilizada en los párrafos finales (40\%); las conclusiones están redactadas de forma impersonal (100\%); y la presencia del objeto de estudio en los párrafos finales es más prevalente a través de la nomenclatura común $(62,50 \%)$. Se concluye que la forma diversificada de presentación de los párrafos finales en los artículos científicos nacionales, en el área de las ciencias agrarias, merece especial atención en la búsqueda de un modelo que permita acotar su forma múltiple para una redacción más funcional.

Palabras clave: Ensenãnza; Metodología científica; Conclusión; Artículo científico.

\section{Introdução}

A Conclusão é, efetivamente, a última parte de um artigo científico. Mesmo considerando as seções Agradecimentos (nem sempre presente) e Referências Bibliográficas (sempre presente), a Conclusão constitui-se na derradeira porção intimamente ligada a toda lógica científica apresentada e revelada em parágrafos anteriores no artigo. Por não existir um modelo universal que determine o formato ideal para a escrita de Conclusão, são variadas as formas de sua escrita em artigos científicos.

Considerando a existência de diferentes estilos de Conclusão, há elementos fundamentais que precisam estar presentes para que esse arremate de uma investigação científica não fomente dúvidas ao leitor. Uma conclusão "inconclusiva" (por ser mal escrita, por exemplo) coloca em xeque todo o trabalho realizado. Nesse sentido, após a labuta da escrita de projeto, instalação e condução do experimento, coleta e análise de dados e a escrita de todo o corpo do artigo, a Conclusão precisa, também, ser tratada com a mesma energia dispensada nas demais seções do artigo. Conclusão é, portanto, o grande desfecho do que foi investigado em meses, anos ou décadas e, desta forma, é necessário se ter disposição para consumar os achados científicos de forma clara, sucinta e, ao mesmo tempo, completa (Aquino, 2010, p. 80). E, sempre é oportuno ter em mente que a objetividade é uma qualidade basilar da escrita científica (Andrade, 2004, p. 120).

Torna-se imperioso que na Conclusão prevaleça a mesma harmonia dos três elementos textuais (introdução, desenvolvimento e conclusão) de um trabalho acadêmico (NBR 14724) durante a escrita de um artigo científico. Geralmente, as informações apresentadas na Conclusão de um paper são mais utilizadas pela comunidade científica, tanto para a aquisição de novas informações quanto para o embasamento teórico de novas pesquisas. Nesse contexto, o autor deve usar essa oportunidade final de declarar o significado de sua pesquisa (Turbek et al., 2016), onde apenas o essencial deve ser apresentado na Conclusão (sem omissões e sem prolixidade). Portanto, no seu desfecho, a Conclusão deve permitir ao leitor lembrar do conteúdo e da importância do artigo (WU, 2021) e deixar a impressão duradoura de suas ideias (USC, 2021).

Entretanto, é perceptível aos olhos da comunidade científica que há divergências na apresentação da Conclusão entre as revistas, tanto pelo lado editorial quanto autoral. A discordância mais comum encontrada na seção conclusiva de um artigo científico, em relação ao formato (editorial), é a sua própria nomenclatura: Conclusão, Conclusões, Considerações Finais etc. Já em relação ao conteúdo conclusivo, há autores que não têm clareza o suficiente para diferenciar conclusão de resultados e, desta forma, a escrita pode ficar repetitiva do que já está escrito na seção Resultados e Discussão. A escrita da Conclusão deve manter a mesma chama da "ordenação e rigor científico", presentes nos Resultados (Braga, 20112) e nas demais seções do artigo científico.

Artigos completos publicados em revistas científicas na área de ciências agrárias demonstram, por exemplo, a ausência de um modelo no uso da escrita de Palavras-chave (Aquino \& Aquino, 2013). Assim, considerando que "toda pesquisa científica conta uma história" (Volpato, 2015), é imperioso que a narrativa da apresentação científica tenha uma maneira balizada de ser apresentada, tanto na proposta sugerida (formato editorial) quanto no padrão da escrita conclusiva apresentada pelo autor. E, ainda, é pertinente lembrar que a Conclusão é uma das primeiras porções do artigo (juntamente com 
o Título e Resumo) observadas pelo leitor e, desta forma, uma Conclusão bem escrita poderá despertar o interesse do leitor em ler a íntegra do artigo e, eventualmente, até gerar uma citação deste artigo (Caramelli, 2012).

Haja vista a controvertida maneira de se apresentar o panorama conclusivo de informações em artigos científicos objetivou-se, com este trabalho, examinar os aspectos da escrita de Conclusão em artigos científicos da área de ciências agrárias.

\section{Material e Métodos}

Foram utilizados 40 artigos científicos da área de ciências agrárias, publicados em 40 revistas científicas nacionais (tanto em português quanto em inglês), no período de 2003-2017 (Tabela 1). A maioria dos artigos utilizados neste estudo refere-se a pesquisas com plantas, os quais apresentam o nome vulgar e/ou científico no Título.

Esses artigos foram coletados por alunos do Programa de Pós-Graduação em Agronomia (PPGA) da Universidade Federal da Paraíba (UFPB). A coleta dos artigos foi realizada durante as aulas da disciplina Metodologia Científica ministradas por este autor. Os critérios de coleta das amostras (artigos) tiveram como base as "ciências agrárias", onde os alunos do PPGA escolheram, de forma espontânea, a fonte de busca de sua conveniência: sites de periódicos ou revistas disponíveis na biblioteca setorial da UFPB. 
Tabela 1. Relação das revistas científicas da área de ciências agrárias utilizadas na análise sobre a escrita de Conclusão.

\begin{tabular}{|c|c|c|c|c|}
\hline & Revista & Ano & Volume & Número \\
\hline 1 & Acta Bot. Bras. & 2007 & 21 & 2 \\
\hline 2 & Agropec. Cient. Semiárido & 2013 & 9 & 1 \\
\hline 3 & Agropecuária Técnica & 2014 & 35 & 1 \\
\hline 4 & Arq. Bras. Med. Vet. Zootec. & 2010 & 62 & 1 \\
\hline 5 & Biosci. J. & 2011 & 27 & 6 \\
\hline 6 & Bragantia & 2014 & 73 & 4 \\
\hline 7 & Cerne & 2010 & 16 & 1 \\
\hline 8 & Ciênc. Agríc. & 2015 & 13 & 1 \\
\hline 9 & Ciênc. Agrotec. & 2006 & 30 & 1 \\
\hline 10 & Ciênc. Rural & 2009 & 39 & 5 \\
\hline 11 & Eng. Agrícola & 2005 & 25 & 2 \\
\hline 12 & Fitopatol. Bras. & 2005 & 30 & 6 \\
\hline 13 & Floresta & 2003 & 33 & 3 \\
\hline 14 & Gaia Scientia & 2015 & 9 & 1 \\
\hline 15 & Gl. Sci. Technol. & 2017 & 10 & 1 \\
\hline 16 & Hort. Bras. & 2016 & 34 & 1 \\
\hline 17 & Nucleus & 2012 & 9 & 2 \\
\hline 18 & Pesq. Agropec. Bras. & 2011 & 46 & 12 \\
\hline 19 & Pesq. Agropec. Trop. & 2011 & 41 & 3 \\
\hline 20 & Pesq. Flor. Bras. & 2016 & 36 & 88 \\
\hline 21 & Rev.Agro@ambiente & 2010 & 4 & 2 \\
\hline 22 & Rev. Árvore & 2017 & 41 & 1 \\
\hline 23 & Rev. Biociên. & 2004 & 10 & 3 \\
\hline 24 & Rev. Biol. Ciên. Terra* & 2005 & 5 & 2 \\
\hline 25 & Rev. Bras. Agric. Irrigada & 2017 & 10 & 7 \\
\hline 26 & Rev. Bras. Agrociência & 2005 & 11 & 4 \\
\hline 27 & Rev. Bras. Agroecologia & 2016 & 11 & 2 \\
\hline 28 & Rev. Bras. Ciênc. Agrárias & 2009 & 4 & 1 \\
\hline 29 & Rev. Bras. Ciênc. Solo & 2008 & 32 & - \\
\hline 30 & Rev. Bras. Eng. Agric. Ambiental & 2012 & 16 & 12 \\
\hline 31 & Rev. Bras. Fisiol. Veg. & 2001 & 13 & 2 \\
\hline 32 & Rev. Bras. Sementes & 2009 & 31 & 3 \\
\hline 33 & Rev. Bras. Zootec. & 2006 & 35 & 3 \\
\hline 34 & Rev. Caatinga & 2015 & 28 & 4 \\
\hline 35 & Rev. Ciênc. Agronômica & 2013 & 44 & 1 \\
\hline 36 & Rev. da FZA* & 2011 & 18 & 2 \\
\hline 37 & Rev. Verde Agroec. Desenv. Sust. & 2015 & 10 & 4 \\
\hline 38 & Sci. Agric. & 2006 & 63 & 5 \\
\hline 39 & Semina: Ciências Agrárias & 2012 & 33 & 2 \\
\hline 40 & Tecnol. \& Ciên. Agropec. & 2014 & 8 & 1 \\
\hline
\end{tabular}

* Revista descontinuada. Fonte: Aquino (2021). 
Os artigos coincidentes apresentados pelos alunos foram eliminados, sendo escolhido apenas um artigo de cada revista científica, independente do ano de publicação, para ser efetuada a análise. A coletânea de artigos cobre um intervalo de tempo de 15 (quinze) anos.

Os parâmetros analisados foram: 1. Formas (sinônimos) da escrita de Conclusão; 2. Tipo de sinal usado antes de cada conclusão; 3. Número de conclusões; 4. Estilo da conclusão; 5 . Tempo de verbo utilizado nos parágrafos conclusivos; 6. Presença de expressões personificadas; e 7. Presença do objeto de estudo nos parágrafos conclusivos. Os resultados foram agrupados, em tabelas, com números reais e percentagem.

\section{Resultados e Discussão}

A Tabela 2 mostra as diferentes formas (sinônimos) da escrita de Conclusão encontradas em artigos científicos publicados em periódicos das ciências agrárias em um período de 15 (quinze) anos. Observa-se, nesta seção, que há 5 (cinco) formas da escrita conclusiva encontradas nos 40 artigos estudados e, ainda, artigos "sem conclusão e com "conclusão oculta" (onde o leitor tenta "garimpar” a conclusão dentro da seção de Resultados e Discussão).

Tabela 2. Formas da escrita de Conclusão encontradas em artigos científicos publicados em periódicos na área de ciências agrárias.

\begin{tabular}{lcc}
\hline \multicolumn{1}{c}{ Formas da escrita } & N & $\%$ \\
\hline Conclusão & 3 & 7,50 \\
CONCLUSÃO/CONCLUSION & 9 & 22,50 \\
CONCLUSÕES/CONCLUSIONS & 13 & 32,50 \\
Conclusões & 6 & 15,00 \\
CONCLUSÕES & 4 & 10,00 \\
- SEM CONCLUSÃO & 4 & 10,00 \\
- CONCLUSÃO OCULTA & 1 & 2,50 \\
\hline TOTAL & $\mathbf{4 0}$ & $\mathbf{1 0 0 , 0 0}$ \\
\hline
\end{tabular}

Fonte: Aquino (2021).

Considerando as duas formas 'singular' da expressão, os termos "Conclusão" e "CONCLUSÃO/CONCLUSION" correspondem a 30\% dos artigos, com 7,50 e 22,50\%, respectivamente. Já para as três formas 'plural' da expressão conclusiva, “CONCLUSÕES/ CONCLUSIONS", "Conclusões" e "CONCLUSÕES”, observa-se um significativo incremento, com 57,50\% das publicações, com 32,50, 15,00 e 10,00\%, respectivamente.

Nas 40 revistas pesquisadas observou-se, em 4 (quatro) dos artigos (12,50\%), a ausência da nomenclatura conclusiva. Nesses casos, tais artigos foram escritos sem conclusão alguma. Desta forma, sem a presença do 'ponto de vista' do autor (Severino, 2007, p. 150) [na Conclusão], restará ao leitor empregar um trabalho extra na garimpagem de informação conclusiva entre as várias sentenças escritas na seção de Resultados e Discussão. Verificou-se, também, a existência de 1 (um) artigo (2,50\%) com conclusão oculta; neste caso, a conclusão foi escrita no último parágrafo da seção Resultados e Discussão, sem a presença de tópico conclusivo. Há quem considere ser pouco relevante se ter a um tópico para a escrita das conclusões. Pereira (2011, p. 127), por exemplo, acredita que adoção de um novo item para esse fim é algo do passado.

Nesse estudo, observou-se que há revistas que apresentam inconsistência (em volumes distintos) na nomenclatura conclusiva. Na Revista Ciência Rural, por exemplo, encontra-se a expressão CONCLUSÕES em um artigo (v. 39, no. 5, ago. 2009) e CONCLUSÃO em outro (v. 41, no. 3, mar. 2011). Essa incongruência (ora singular, ora plural) é algo que foge do escopo de quem submete um manuscrito para publicação; é algo de competência editorial. 
Além das (cinco) expressões conclusivas encontradas nestas revistas da área de ciências agrárias aqui apresentadas, é comum encontrar artigos científicos com outras nomenclaturas terminativas: Considerações Finais, Considerações Gerais, À Guisa de Conclusão; estas, são encontradas com mais facilidade em artigos da área de ciências humanas. $\mathrm{O}$ autor, ao se deparar com a necessidade de submeter um manuscrito a uma revista que adota Considerações Finais, por exemplo, precisa sempre ter em mente a necessária adoção de objetividade; caso contrário, o texto conclusivo, nesses moldes, ficará difuso. Assim, para evitar uma conclusão indefinida, torna-se necessário que o autor deixe claro em seu texto se os objetivos foram alcançados (Pereira et al., 2018).

Quando a revista utiliza o termo Conclusão (singular e em suas mais variadas formatações editoriais), o autor pode escrever a(s) sua(s) conclusão(sões) e a leitura tornar-se algo 'confortável' (a singularidade do vocábulo "Conclusão" poderá abrigar uma ou mais conclusões nessa seção). Entretanto, quando a revista adota o termo Conclusões (plural e em suas mais variadas formatações editoriais), a leitura não fica 'confortável' quando, por exemplo, o autor tem apenas UMA conclusão a apresentar (ver Lulu et al., 2005). Em síntese, a escrita da Conclusão de um artigo científico deve deixar um sentimento de satisfação ao leitor (Aquino, 2012, p. 59), tanto por sua estrutura editorial quanto do conteúdo autoral.

Em relação ao tipo de sinal usado antes de cada conclusão em artigos científicos publicados em periódicos na área de ciências agrárias, constata-se (Tabela 3) que a adoção de parágrafos simples predomina $(70,00 \%)$ a forma de apresentar as conclusões.

Tabela 3. Tipo de sinal usado antes de cada conclusão em artigos científicos publicados em periódicos na área de ciências agrárias.

\begin{tabular}{lrr}
\hline \multicolumn{1}{c}{ Tipo de sinal } & N & \multicolumn{1}{c}{$\%$} \\
\hline Parágrafo(s) simples & 28 & 70,00 \\
Parágrafo(s) com número & 6 & 15,00 \\
Parágrafo(s) com símbolo ou forma geométrica & 1 & 2,50 \\
- Sem parágrafo(s) conclusivo(s)* & 5 & 12,50 \\
\hline TOTAL & $\mathbf{4 0}$ & $\mathbf{1 0 0 , 0 0}$ \\
\hline
\end{tabular}

* 4 artigos sem conclusão; 1 artigo com conclusão oculta. Fonte: Aquino (2021).

Observa-se que o uso de parágrafos conclusivos precedidos de números (1., 2., ...), 15\%; e símbolo [•] ou forma geométrica $(2,5 \%)$ não são tão preferidos pelas revistas agronômicas. O uso de número, por exemplo, mesmo com baixa preferência nos artigos pesquisados neste estudo, expressam uma maior didática em relação ao parágrafo simples ou ao uso de símbolos. É mais fácil pontuar "por favor, olhe a conclusão 3" do que mencionar "parágrafo terceiro" ou "bolinha número três". Além disso, é comum encontrar revistas em que utilizam hífen (-) ou outros símbolos, geométricos (•) ou não $(\checkmark,>$, *), no início de cada parágrafo conclusivo.

O número de conclusões específicas presentes em artigos científicos (Tabela 4) publicados em periódicos na área de ciências agrárias mostra a predominância de duas conclusões por artigo (45\%), seguidos por publicações que apresentam apenas uma (17\%), três (15\%), quatro $(7,5 \%)$ ou cinco $(2,5 \%)$ conclusões. 
Tabela 4. Número de conclusões específicas presentes em artigos científicos publicados em periódicos na área de ciências agrárias.

\begin{tabular}{|c|c|c|}
\hline Número de conclusões & $\mathbf{N}$ & $\%$ \\
\hline Uma & 7 & 17,50 \\
\hline Duas & 18 & 45,00 \\
\hline Três & 6 & 15,00 \\
\hline Quatro & 3 & 7,50 \\
\hline Cinco & 1 & 2,50 \\
\hline - Sem parágrafo(s) conclusivo(s)* & 5 & 12,50 \\
\hline TOTAL & 40 & 100,00 \\
\hline
\end{tabular}

Fonte: Aquino (2021).

A Tabela 5 exibe o estilo da conclusão presente em artigos científicos publicados em periódicos na área de ciências agrárias. Observa-se que quase $20 \%$ dos artigos apresentam conclusões com informações adicionais: recomendação, $10 \%$; justificativa, 5\%; resultado, 2,5\%. Essas informações complementares já possuem o seu local específico: a seção Resultados e Discussão; desta forma, não convém adicionar qualquer outra informação, ideia ou argumento além do texto conclusivo. Qualquer referência a algo novo, que não esteja relacionado ao objetivo da pesquisa, deve ser evitado nesta seção, com ressalva a alguma proposta do autor (Matias-Pereira, 2007, p. 99) para investigações adicionais, por exemplo, a qual deve ser escrita no último parágrafo da Conclusão.

Tabela 5. Estilo da conclusão presente em artigos científicos publicados em periódicos na área de ciências agrárias.

\begin{tabular}{lcr}
\hline \multicolumn{1}{c}{ Estilo da conclusão } & N & \% \\
\hline Apenas a Conclusão (sem informação adicional) & 28 & 70,00 \\
Conclusão com recomendação & 4 & 10,00 \\
Conclusão com justificativa & 2 & 5,00 \\
Conclusão com resultado & 1 & 2,50 \\
- Sem parágrafo(s) conclusivo(s)* & 5 & 12,50 \\
\hline TOTAL & $\mathbf{4 0}$ & $\mathbf{1 0 0 , 0 0}$ \\
\hline
\end{tabular}

* 4 artigos sem conclusão; 1 artigo com conclusão oculta. Fonte: Aquino (2021).

Seguindo uma das regras basilares da escrita científica, é importante que a Conclusão seja escrita com frases breves (Fioreze, 2003, p. 66; Fachin, 2017, p.186). E essa regra é aplicada tanto em trabalhos de graduação, quanto em monografias da pós-graduação (Ferreira et al., 2020) e até em apresentação de seminários (Marconi \& Lakatos, 2009, p.35; Aquino, 2010, p. 22). Cumpre observar, ainda, que uma Conclusão é escrita para ser lida e, portanto, é indispensável que sua redação seja coesa com o objetivo do artigo (Pereira, 2012) e, ao mesmo tempo, "breve, exata, concisa e convincente" (Andrade, 2004, p.120).

Quanto ao tempo de verbo utilizado nos parágrafos conclusivos em artigos científicos publicados em periódicos na área de ciências agrárias (Tabela 6), percebe-se que a maioria das publicações emprega o tempo de verbo no tempo presente $(40,00 \%)$, enquanto que $32,50 \%$ dos artigos apresenta os parágrafos conclusivos no tempo pretérito. 
Tabela 6. Tempo de verbo utilizado nos parágrafos conclusivos em artigos científicos publicados em periódicos na área de ciências agrárias

\begin{tabular}{lrc}
\hline \multicolumn{1}{c}{ Tempo de verbo na Conclusão } & N & \% \\
\hline Presente & 16 & 40,00 \\
Pretérito & 13 & 32,50 \\
Presente e Pretérito & 7 & 17,50 \\
- Sem parágrafo(s) conclusivo(s)* & 4 & 10,00 \\
\hline TOTAL & $\mathbf{4 0}$ & $\mathbf{1 0 0 , 0 0}$ \\
\hline
\end{tabular}

* Não avaliados por não possuir a seção Conclusão/Conclusões; foi avaliado apenas o único artigo com conclusão oculta (presente no último parágrafo de Resultados e Discussão. Fonte: Aquino (2021).

O emprego da conclusão no tempo de verbo presente tende a fortalecer os "achados" (que estão sendo publicados), uma vez que fica - de certa forma - subentendido que se alguém repetir a mesma pesquisa (nas mesmas condições), haverá grande probabilidade de se encontrar os mesmos resultados. Já o uso do tempo de verbo no pretérito deixa margem para se pensar: “... funcionou assim no passado, mas não deve funcionar assim no presente”. Porém, é importante ponderar a percepção de Volpato (2015) onde, em situações que tenha havido algum "fenômeno" importante, uma conclusão pode ser escrita no tempo pretérito.

De forma estupefaciente, encontrou-se 17,50\% dos artigos com o uso incorreto do tempo de verbo das conclusões: uma conclusão no presente, outra no pretérito. Nestes casos, a intermitência do tempo de verbo macula todo texto científico apresentado até então.

A Tabela 7 expõe o contraste da impessoalidade textual com textos conclusivos personificados. A forma impessoal na escrita conclusiva apresentou-se como forma padrão (100\% dos artigos). Esta característica apresentada na seção conclusiva do artigo deve estar presente em todo o corpo do artigo científico.

Tabela 7. Presença de expressões personificadas utilizadas nos parágrafos conclusivos em artigos científicos publicados em periódicos na área de ciências agrárias.

\begin{tabular}{lrc}
\hline \multicolumn{1}{c}{ Não personificada versus Personificado } & N & \% \\
\hline Não personificada (forma impessoal) & 36 & 100,00 \\
Personificada & 0 & 00,00 \\
- Sem parágrafo(s) conclusivo(s)* & 4 & 10,00 \\
\hline TOTAL & $\mathbf{3 6}$ & $\mathbf{1 0 0 , 0 0}$ \\
\hline
\end{tabular}

* Não analisados: 4 artigos sem conclusão; analisado: 1 artigo com conclusão oculta. Fonte: Aquino (2021).

É oportuno observar que a unanimidade apresentada no texto conclusivo das publicações estudadas não pode servir de analogia aos textos escritos previamente no artigo científico (Introdução, Objetivo, Material e Métodos, Resultados e Discussão). Muitas vezes, é possível encontrar verbos que apresentam afirmações personificadas (principalmente na $3^{\mathrm{a}}$ pessoa do plural: "realizamos", "aplicamos", "colhemos" etc.) em outras seções do artigo científico.

O texto conclusivo, preferencialmente, deve ser escrito de forma impessoal, bem como todos os elementos textuais (todas as seções) de um artigo científico completo. Além da impessoalidade, Gil (2009) alerta para outras qualidades essenciais da redação científica em projetos como objetividade, clareza, precisão, coerência, concisão e simplicidade (p.164-165) que, de forma semelhante, se aplicam a escrita científica como um todo. Há, entretanto, controvérsia em relação a personificação da escrita (Volpato, 2015). 
Uma vez que 97,50\% dos trabalhos avaliados tinham um componente vegetal (gramínea, arbusto, árvore) como foco da pesquisa, examinou-se a presença da nomenclatura (vulgar e/ou científica) do objeto de estudo nos parágrafos conclusivos (Tabela 8).

Tabela 8. Averiguação da presença do objeto de estudo nos parágrafos conclusivos em artigos científicos publicados em periódicos na área de ciências agrárias.

\begin{tabular}{lrr}
\hline \multicolumn{1}{c}{ Presença do objeto de estudo na Conclusão } & N & \multicolumn{1}{c}{$\%$} \\
\hline Apenas a nomenclatura cientifica completa (Género espécie) & 3 & 7,50 \\
Apenas a nomenclatura científica abreviada (G. espécie) & 3 & 7,50 \\
Nome vulgar seguido de nome cientifico & 1 & 2,50 \\
Apenas a nomenclatura vulgar & 25 & 62,50 \\
Sem nomenclatura científica e nem vulgar & 3 & 7,50 \\
Não se aplica & 1 & 2,50 \\
-Sem parágrafo(s) conclusivo(s) & 4 & 10,00 \\
\hline TOTAL & $\mathbf{4 0}$ & $\mathbf{1 0 0 , 0 0}$ \\
\hline
\end{tabular}

* Não analisados: 4 artigos sem conclusão; analisado: 1 artigo com conclusão oculta. Fonte: Aquino (2021).

Vale ressaltar que este estudo não se deteve a quantificar o número de trabalhos com erros na apresentação da nomenclatura científica presentes, nem tampouco a qualificação desses erros (Além disso, não se levou em consideração a presença (ou não) de identificador e/ou data da identificação). Observa-se que a presença da nomenclatura científica nos parágrafos conclusivos se encontra em apenas $15 \%$ dos artigos (7,50\% com a nomenclatura científica completa; 7,50\% com a nomenclatura científica abreviada). Apenas 1 (um) artigo (2,50\%) apresentou nomenclatura vulgar, seguida da nomenclatura científica.

Surpreendentemente, $62,50 \%$ dos artigos têm em seus parágrafos conclusivos apenas a nomenclatura vulgar. Esta é, sem dúvida, uma omissão que não se justifica, principalmente por tratar-se do desfecho de uma investigação científica o qual, necessariamente, carece da precisa identificação dos aspectos conclusivos relacionados ao organismo estudado.

Outro achado inverossímil é encontrar artigos sem nomenclatura científica e nem vulgar (7,50\%). Mesmo sendo uma quantidade reduzida, textos conclusivos desta natureza constituem-se em "conclusões inconclusivas" e, portanto, injustificáveis. É oportuno, ainda, o registro de que há trechos conclusivos em que há inconsistência na apresentação de nomes científicos. Um dos exemplos emblemáticos é quando se encontra, no mesmo parágrafo conclusivo, um nome científico de uma espécie "A" seguido de apenas um nome vulgar para uma espécie "B"; ou seja, a aplicação de nomenclatura científica para apenas um ser vivo e vulgar para outro.

$\mathrm{Na}$ seção conclusiva de um artigo científico é imprescindível que o autor responda às questões propostas em sua pesquisa e discutidas anteriormente (Vergara, 2010, p. 80). Portanto, nada mais óbvio do que o resgate da nomenclatura científica, mesmo que já anteriormente citada em qualquer (ou todas) seção preliminar (título, objetivo, introdução, metodologia, resultados e discussão). A parte conclusiva deve, desta maneira, deixar explícito um arremate compatível aos objetivos e hipóteses propostas (NBR 6022) na parte introdutória do artigo científico. Esta observação aplica-se tanto ao(s) autor(es) quanto a editor(es) e revisores/consultores ad hoc pois, ao final, o profissionalismo na apresentação do artigo (Aquino \& Aquino, 2013) será compartilhado por todos os envolvidos.

A desarmonia no formato da escrita científica, nas 40 revistas analisadas (publicadas em um período de 15 anos), mostra a necessidade de uma avaliação mais cuidadosa por parte dos avaliadores das revistas em relação à escrita dos 
parágrafos conclusivos dos artigos; isso sem ignorar a necessidade de qualificar melhor alunos de graduação e (principalmente) pós-graduação, enquanto cursam a disciplina de metodologia científica e são treinados para exercerem o papel de cientistas. Com um comprometimento de todos os partícipes na melhor escrita de parágrafos conclusivos em artigos científicos na área de ciências agrárias, eventualmente esses erros e omissões serão diluídos com novas publicações.

\section{Conclusão}

Este estudo sobre a forma da escrita conclusiva em artigos científicos nacionais na área de ciências agrárias, publicados durante o período de 2003-2017, permite tirar as seguintes conclusões:

1. Não há uma uniformização (editorial e autoral) na escrita de parágrafos conclusivos em artigos científicos na área de ciências agrárias;

2. CONCLUSÕES (plural e em caixa alta) é a maneira (editorial) mais usada como a 'chamada' da seção conclusiva nas revistas analisadas;

3. Parágrafo simples (sem número ou símbolo) é predominante nos artigos publicados;

4. A maioria dos artigos apresenta apenas (duas) conclusões específicas;

5. Prevalece - de forma correta - a exibição apenas da Conclusão (sem informação adicional);

6. O tempo de verbo no "presente" é a forma mais utilizada nos parágrafos conclusivos;

7. Há hegemonia em conclusões escritas de forma impessoal; e

8. A presença do objeto de estudo (organismo vegetal) nos parágrafos conclusivos tem predomínio de uso da nomenclatura vulgar.

Torna-se necessário análises adicionais sobre a forma da escrita científica de outras seções em artigos, de revistas indexadas das ciências agrárias, para um melhor entendimento do perfil dessas publicações.

\section{Agradecimentos}

O autor agradece a colaboração dos alunos de pós-graduação da disciplina Metodologia da Pesquisa Científica, Programa de Pós-Graduação em Agronomia (PPGA) da Universidade Federal da Paraíba (UFPB), pelo fornecimento dos artigos científicos utilizados nesta pesquisa.

\section{Referências}

Andrade, M. M. (2004). Como preparar trabalhos para cursos de pós-graduação. (6a ed.), Atlas, 165 p.

Aquino, I. S. (2010). Como escrever artigos científicos - sem arrodeio e sem medo da ABNT. (7a ed.), Saraiva, 126 p.

Aquino, I. S. (2010). Como falar em encontros científicos - do seminário em sala de aula a congressos internacionais. $5^{\text {a }}$ ed. São Paulo: Saraiva, 110 p.

Aquino, I. S. (2010). Como ler artigos científicos - da graduação ao doutorado. (3a ed.), Saraiva, 94 p.

Aquino, I. S. \& Aquino, I. S. (2013). Análise sobre a forma da escrita de palavras-chave em artigos científicos na área de ciências agrárias publicados no período de 1999 a 2011. Revista Brasileira de Biblioteconomia e Documentação, 18(37), 227-238, https://doi.org/10.5007/1518-2924.2013v18n37p227

Associação Brasileira De Normas Técnicas (ABNT). (2003). NBR 6022: Informação e documentação: artigo em publicação periódica científica impressa: apresentação. $25 \mathrm{p}$.

Associação Brasileira De Normas Técnicas (ABNT). (2011). NBR 14724: Informação e documentação: trabalhos acadêmicos: apresentação. 11 p.

Braga, A. L. F. (2012). Resultados - “chegou a hora desta gente bronzeada mostrar seu valor”. Revista da Associação Médica Brasileira, 58(4), 1-2. https://doi.org/10.1590/S0104-42302012000400003

Caramelli, B. (2012). Conclusão: como exibir a cereja do bolo. Revista da Associação Médica Brasileira, 58(6), 633. 
Research, Society and Development, v. 10, n. 5, e58410515206, 2021

(CC BY 4.0) | ISSN 2525-3409 | DOI: http://dx.doi.org/10.33448/rsd-v10i5.15206

Fachin, O. (2017). Fundamentos de metodologia científica - noções básicas em pesquisa científica. (6a ed.), Saraiva, 200 p.

Ferreira, A. C. S.; Ranieri, M. G. A; Capellato, P.; Silva, G. \& Silva, L. F. (2020). Como escrever uma dissertação de mestrado. Pesquisa, Sociedade e Desenvolvimento, 9(3), e149932584. 10.33448 / rsd-v9i3.2584. https://rsdjournal.org/index.php/rsd/article/view/2584.

Fioreze, R. (2003). Metodologia da pesquisa - como planejar, executar e escrever um trabalho científico. (2a ed.), Ed. Universitária UFPB, 144 p.

Gil, A. C. (2009). Como elaborar projetos de pesquisa. Atlas, 1754 p.

Lulu, J., Castro, J. V. \& Pedro Jr., M. J. (2005). Armazenamento refrigerado de uva de mesa 'romana' (A1105) cultivada sob cobertura plástica. Engenharia Agrícola. 24(2), 481-487. https://doi.org/10.1590/S0100-69162005000200022

Marconi, M. A. \& Lakatos, E. M. (2009). Metodologia do trabalho científico: procedimentos básicos, pesquisa bibliográfica, projeto e relatório, publicações e trabalhos científicos. Atlas, 225 p.

Matias-Pereira, J. (2007). Manual de metodologia da pesquisa científica. Guanabara Koogan, 384 p.

Pereira, M. G. (2011). Artigos científicos: como redigir, publicar e avaliar. Atlas, 151 p.

Pereira, M. G. (2012). Preparo para a redação do artigo científico. Epidemiologia e Serviços de Saúde, 21(3), 227-238, http://dx.doi.org/10.5123/S167949742012000300017

Pereira, A. S., Shitsuka, D. M., Parreira, F. J. \& Shitsuka, R. (2018). Metodologia da pesquisa científica. UFSM, 119 p. https://repositorio.ufsm.br/bitstream /handle/1/15824/Lic_Computacao_Metodologia-Pesquisa-Cientifica.pdf?sequence=1\%205.4)

Severino, A. J. (2007). Metodologia do trabalho científico. (23a ed.), Cortez, 304 p.

Turbek, M. C., Chock, T. M., Donahue, K., Havrilla, C., Oliverio, A. M., Polutchko, S. K., Shoemaker, L. G. \& Vimercati, L. (2016). Scientific writing made easy: a step- by- step guide to undergraduate writing in the biological sciences. The Bulletin of the Ecological Society of America, 97(4), 417-426. https://doi.org/10.1002/bes 2.1258

University of Southern California - USC. (2021). Organizing Your Social Sciences Research Paper. https://libguides.usc.edu/writin gguide/conclusion

Vergara, S. C. (2010). Projetos e relatórios de pesquisa em administração. Atlas, 94 p.

Volpato, G. L. (2015). O método lógico para redação científica. Revista Eletrônica de Comunicação, Informação \& Inovação em Saúde, 9 (1), 1-14. https://doi.org/10.29397/reciis.v9i1.932

Walden University - WU. (2021). Writing a paper: conclusions. https://academicguides.waldenu.edu/writingcenter/writingprocess/conclusions 